\title{
Phytochemical Characterization of Alternanthera sessilis and Assessment of its Growth Promoting Potential on the Freshwater Prawn Macrobrachium rosenbergii
}

\author{
Rathinam Kanagarasu ${ }^{1}$, Periyakali Saravana Bhavan ${ }^{1}$, Gopalan Rajkumar ${ }^{1}$, \\ Virumandi Nathiya $^{1}$, Thangavelu Satgurunathan ${ }^{1}$, Thangaraj Manjula ${ }^{1}$ \\ ${ }^{1}$ Department of Zoology, Bharathiar University, Coimbatore - 641046, Tamil Nadu, India
}

*Corresponding Author: Periyakali Saravana Bhavan, ${ }^{1}$ Department of Zoology, Bharathiar University, Coimbatore, Tamil Nadu, India

\begin{abstract}
This study was aimed to understand the primary and secondary phytochemical profiles of Alternanthera sessilis and its influence on growth promotion of Macrobrachium rosenbergii. Complete extraction of A. sessilis leaf using petroleum ether (non-polar), followed by acetone (middle polar) and ethanol (polar) was performed first. Then the primary and secondary phytochemical profiles were studied. A. sessilis contains alkaloids, terpenoids, flavonoids, tannins, saponins, polyphenols, cardiac glycosides and quinones. GC-MS spectrum of A. sessilis revealed the presence of 17 secondary phytochemicals, totally. Among these, 5 compounds have possessed bioactive properties. Artificial feeds were prepared with incorporation of three different concentrations (0.1, 0.5 and 1\%) of ethanolic extract of A. sessilis and fed to M. rosenbergii PL for 90 days. Among the three levels, $1.0 \%$ of ethanolic extract of A. sessilis incorporated feed produced the best growth performance, by showing the highest survival rate, weight gain and contents of total protein, amino acid, carbohydrate, lipid and ash, when compared with control, which was confirmed by the lowest food conversion rate. Thus, the present work recommends incorporation of A. sessilis as feed additive to attain sustainable culture of $M$. rosenbergii.
\end{abstract}

Keywords: Herbal extraction, Active principles, Prawn culture.

\section{INTRODUCTION}

India is making rapid strides with its blue revolutions and holds second position in the world in aquaculture production, producing $7.76 \%$ in 2013, yet far below from China, which holds number one position with almost $61.35 \%$ production. Crustacean, which includes crabs, lobsters, crayfish, prawns, shrimps etc. consisted of 50,000 to 67,000 species and has become one of the fastest growing animal production sectors worldwide. Among these about 4048 species of shrimps and prawns are known to the world (Radhakrishnan et al., 2012).

In India, the freshwater prawn fishery of the rivers Krishna, Godavari, Parkas, Ganges, Mahanadi, Hooghly and Cauvery indicate that the major commercial species are Macrobrachium rosenbergii and Macrobrachium malcolmsonii. Their aquacultures have become more popular due to nutritious delicacy for mankind, and earn valuable foreign exchange because of good demand in the international markets. $M$. rosenbergii has shorter life history than that of marine shrimps, and it is a fast growing species, reaches considerable size within short period under intensive culture. The nutritive value of any crustacean depends up on its protein, amino acid, lipid, fatty acid, carbohydrate, vitamins and minerals (Bhavan et al., 2010). Other Palemonid prawns, such as Macrobrachium nobilii, Macrobrachium lamarrei, Macrobrachium scabriculum, Macrobrachium birmanicumchoprai, Macrobrachium mirable, Macrobrachium rude, Macrobrachium hendersonii, Macrobrachium villosimanus and Macrobrachium hendersonii also support local fisheries (Chandrasekaran and Sharma, 1997; Mariappan et al., 2003).

Feed is the major operational cost for most aquaculture enterprises (D'Abramo and Sheen, 1994). The formulation of well-balanced diets and their adequate feeding are the most important for successful aquaculture. The formulation of feeds by using locally available low cost agricultural and animal husbandry byproducts (such as soy bean meal, rice brawn, wheat brawn and ground nut oilcake, 
fishmeal, poultry waste, blood meal and prawn meal etc.) have crucial role in aquaculture industry (Mitra et al., 2005; Langer et al., 2011).

Currently, researchers have focused on herbal plants, greens, algae, vegetable and fruit wastes as low cost food supplements for better growth and survival of Macrobrachium (Rebecca and Bhavan, 2011; Shanthi et al., 2012; Bhavan and Radhakrishnan, 2012; Radhakrishnan et al., 2013; Aarumugam et al., 2013; Bhavan et al., 2011, 2012, 2013a,b, and 2014a,b; Dhanalakshmi et al., 2016; Rajkumar et al., 2017). In this line, Alternanthera sessilis a common pan-tropical weed of shady, damp soils in cultivable and waste land has been chosen for screening of its phytochemicals, and whether can it be used as a feed additive for Macrobrachium culture. A. sessilis is a popular leafy vegetable, and is used as a folk medicine in Southeast Asia. For example, the juice of this plant is an ingredient in medicinal hair oils (Gupta, 2014). It serves as good fodder as well. The genus, Alternanthera has possessed anti-inflammatory, antimicrobial, antiviral, antitumor, anti-malarial, and anti-ulcer properties (Guerra et al., 2003; Hilou et al., 2006). In this study, A. sessilis leaf was subjected to extraction with three different solvents, such as petroleum ether, acetone and ethanol for determining its phytochemicals. The ethanolic extract of $A$. sessilis was incorporated with artificial feed formulated, and its growth promoting potential was assessed on M. rosenbergii PL.

\section{Materials AND Methods}

\subsection{Preparation of $A$. sessilis Leaf Extract}

The medicinal herb, A. sessilis was collected from Bharathiar University campus, Coimbatore, Tamil Nadu, India. The herb was thoroughly washed with freshwater, blotted and spread out and dried for two weeks at room temperature. Shade dried herb was ground to fine powder. A. sessilis powder (50 g) was packed with Whatmann No. 1 filter paper and put into Soxhlet apparatus, successively and sequentially extracted with $300 \mathrm{ml}(1: 6 \mathrm{w} / \mathrm{v})$ of individual solvent (petroleum ether, acetone and ethanol) for 6-9 h each (30 to 36 cycles) based on the polarity (non-polar to polar) until a clear colorless solution was obtained. The extract obtained was filtered by using double layer muslin cloth, and concentrated at $40-50^{\circ} \mathrm{C}$ using rotary vacuum evaporator (ROTAVAP) with ultra-cryostat and dried at $40^{\circ} \mathrm{C}$ under hot air oven. The dark green, gummy solid obtained was used for further investigation.

\subsection{Qualitative Analysis of Phytochemicals}

The presence of primary phytochemical compounds, such as presence of alkaloids, terpenoids, flavonoids, tannins, polyphenols, saponins, cardiac glycosides and quinones was screened by adopting the standard qualitative procedures (Trease and Evans, 1989).

\subsection{Gas Chromatography-Mass Spectrometry (GC-MS) Analysis}

Each extract of A. sessilis was subjected to GC-MS (The Trace GC Ultra and DSQII model MS with inbuilt pre-filter to reduce the neutral particles, Thermo Fisher Scientific Company Pvt. Ltd.) analysis for identification of different secondary phytochemical compounds with following working conditions [Injector port temperature: $250{ }^{\circ} \mathrm{C}$; Interface temperature: $250{ }^{\circ} \mathrm{C}$, and source was maintained at 200 ${ }^{\circ} \mathrm{C}$; The oven temperature: programmed as variable, $70{ }^{\circ} \mathrm{C}$ for $2 \mathrm{mins}, 150{ }^{\circ} \mathrm{C} @ 8{ }^{\circ} \mathrm{C} / \mathrm{min}$, up to 260 ${ }^{\circ} \mathrm{C} @ 10{ }^{\circ} \mathrm{C} / \mathrm{min}$; injector used was splitless mode; Column: The DB-35 MS Nonpolar (Agilent Co., USA) with dimensions of $0.25 \mathrm{~mm}$ OD x $0.25 \mu \mathrm{m}$ ID x 30 metres length; Carrier gas: Helium was used at $1 \mathrm{~mL} / \mathrm{min}$; Scan: 50-650 Da; Motor vacuum pressure: <40; Ionization energy: -70eV].

Peaks resolved with relative abundance of 0-100 were considered as major compounds. To show the minor peaks, the chromatogram was magnified. Identification of various components present in each extract was done by comparison of retention indices and mass spectra fragmentation patterns with those stored on the computer library and also with published literatures. National Institute Standard and Technology (NIST4) and WILEY9 (Vandendool and Kratz, 1963) on-line library source was also used for matching the identified components.

\subsection{Preparation of Feeds}

The following branded feed basal ingredients (BI) were used to formulate the experimental feed. For protein source fish meal (25\%), groundnut oilcake (25\%) and soybean meal $(25 \%)$ were taken. For carbohydrate source wheat bran (10\%) was taken. For lipid source Sunflower oil (2\%) was taken. 
Tapioca flour (5\%) and egg albumin (7\%) were used as binding agents. The powdered basal ingredients such as fish meal, groundnut oilcake, soy bean meal, wheat bran were thoroughly mixed. Then sterilized water was used to prepare the dough, which was steam cooked and cooled at room temperature. Then Sunflower oil was added with the dough and mixed well. The ethanolic extract of A. sessilis was prepared freshly and incorporated with the dough at three different concentrations $0.1 \%, 0.5 \%$ and $1.0 \%$. Then tapioca flour and egg albumin were added and mixed well. Finally, $1 \%$ of vitamin B-complex forte with vitamin C (BECOSULES® CAPSULES, Pfizer Ltd., Navi Mumbai, India) and a pinch of salt (Approximately, 100mg) were also added and thoroughly mixed. Double distilled water $\left(\mathrm{ddH}_{2} \mathrm{O}\right)$ was adequately added for maintaining the mixer in moist and paste form. This was pelletized in a manual pelletizer fixed with $3 \mathrm{~mm}$ diameter mesh. The pellets were immediately dried in a thermostatic oven at $37-40{ }^{\circ} \mathrm{C}$ for one hour to quickly reduce the moisture in order to keep them intact, and then shade dried until they reached constant weight. To maintain its brittleness and prevent fungal attack they were kept in air tight jars, stored at $-20^{\circ} \mathrm{C}$ and used afresh. The proximate composition of organic matters present in the basal diet formulated was determined by adopting the methodology of Castell and Tiews (1980) as given in AOAC (1995) manual, which contains 40.50\% crude protein, $5.60 \%$ crude fat, $3.40 \%$ crude fibre, $9.00 \%$ total ash, $8.60 \%$ moisture, $32.90 \%$ carbohydrate (total nitrogen free extract) and gross energy, $4281 \mathrm{kcal} / \mathrm{kg}$.

\subsection{Feeding Trial}

The post larvae (PL-30) of the freshwater prawn, Macrobrachium rosenbergii were procured from nursery pond at Singanallur (Lat. $10.99^{\circ} \mathrm{N}$; Lon. $77.02^{\circ} \mathrm{E}$ ), Coimbatore, Tamil Nadu, India. They were transported to the laboratory in polythene bags filled with oxygenated water. They were acclimatized to ambient laboratory conditions for 2 weeks (at that time it reached PL-45) in cement tank $(6 \times 3 \times 3$ feet) with ground water (temperature, $27 \pm 1.0{ }^{\circ} \mathrm{C} ; \mathrm{pH}, 7 \pm 0.14$; total dissolved solids, $0.9 \pm 0.006 \mathrm{~g} / \mathrm{l}$; dissolved oxygen, $7.2 \pm 0.55 \mathrm{mg} / \mathrm{l}$; BOD $30.0 \pm 1.28 \mathrm{mg} / \mathrm{l}$; COD, $125.0 \pm 3.1 \mathrm{mg} / \mathrm{l}$; ammonia, $0.028 \pm 0.005 \mathrm{mg} / \mathrm{l})$. During acclimatization the prawns were fed with boiled egg albumin and artificially formulate feed of our own. More than 50\% of tank water was routinely renewed every day in order to maintain a healthy environment and adequate aeration was also provided. This was to ensure sufficient oxygen supply to the prawns and an environment devoid of accumulated metabolic wastes. The unfed feeds, faeces, moult and dead prawns if any were removed by siphoning without disturbing the prawns.

Four groups of $M$. rosenbergii PL-45 (2.63 $\pm 0.11 \mathrm{~cm}$ in length and $0.15 \pm 0.02 \mathrm{~g}$ in weight) each with 30 individuals were maintained in $30 \mathrm{~L}$ plastic tanks in a triplicate experimental set-up. They were starved for $24 \mathrm{~h}$ before beginning of the feeding trial. One group served as control and fed with feed formulated by using BI only, and the other three groups were fed with experimental feeds prepared by incorporation of ethanolic extract of A. sessilis $(0.1 \%, 0.5 \%$ and $1.0 \%)$. The feed was allocated to the prawns for two times a day (8:00 and 20:00 hrs) at 10\% of body weight. The experiment was extended for a period of 90 days, at that time the prawns reached juvenile stage. The unfed feed, feces and moult if any, were collected on daily basis by siphoning with minimum disturbance to the prawns while renewing the aquarium water. For morphometric and nutritional analysis 10 prawns from each group were randomly measured and the mean was considered as a single value (mean of 10 individual measurements $=$ one observation), and three such observations were made to fulfill the triplicate analysis.

\subsection{Evaluations of Nutritional Indices}

After 90 days of feeding trial, the growth parameters, such as survival rate (SR), length gain (LG), weight gain (WG), specific growth rate (SGR), protein efficiency ratio (PER) and food conversion ratio (FCR) were determined by following equations of Tekinay and Davies (2001). Survival rate, SR $(\%)=$ Total No. of live prawn / Total No. of prawns introduced initially $\times 100$. Length gain, LG $(\mathrm{cm})$ $=$ Final length $(\mathrm{cm})-$ Initial length $(\mathrm{cm})$. Weight gain, WG $(\mathrm{g})=$ Final weight $(\mathrm{g})-$ Initial weight $(\mathrm{g})$. Specific growth rate, SGR $(\%)=\log \mathrm{w} 2-\log \mathrm{w} 1 / \mathrm{t} \times 100$ (where, w1 \& w2 represents initial and final weight $(\mathrm{g})$ respectively, and, ' $\mathrm{t}$ ' is the total number of experimental days). Protein efficiency ratio, PER $(\mathrm{g})=$ Weight gain $(\mathrm{g}) /$ Protein intake. Feed conversion ratio, FCR $(\mathrm{g})=$ Total quantity of feed intake $(\mathrm{g}) /$ Weight gain of the prawn $(\mathrm{g})$. 
Phytochemical Characterization of Alternanthera sessilis and Assessment of its Growth Promoting Potential on the Freshwater Prawn Macrobrachium rosenbergii

\subsection{Estimations of Biochemical Constituents}

On the final day of the experiment, the contents of basic biochemical constituents such as total protein (Lowry et al., 1951), total carbohydrate (Roe, 1955), total lipid [extracted by using chloroformmethanol mixture (Folch et al., 1957) and estimated by following the method of Barnes and Blackstock (1973)], ash and moisture (APHA 2005) of individual diet fed prawns were estimated.

\subsection{Statistical Analysis}

The data were expressed as mean $\pm \mathrm{SD}$, and analyzed by one-way analysis of variance (ANOVA) using SPSS (v20), and subsequent post hoc multiple comparison, Duncan's multiple range test (DMRT) to compare the significant differences among treatments at $p<0.05$.

\section{RESUlts}

\subsection{Primary Phytochemicals of $A$. sessilis}

The petroleum etheric leaf extract of A. sessilis showed presence of 6 primary compounds, such as alkaloids, terpenoids, flavonoids, tannins, polyphenols and quinones. Of which alkaloids, terpenoids and polyphenols were moderately present. Flavonoids, tannins and quinones were poorly present (Table 1). In acetonic extract of A. sessilis showed presence of 7 compounds. Of which polyphenols and cardiac gylcosides were luxuriantly present. Tannins and saponins were moderately present. The other compound, such as terpenoids, flavonoids and quinones were poorly present (Table 1). Similarly, ethanolic extract of A. sessilis contained 6 primary compounds, such as, flavonoids tannins, polyphenols, saponins, cardiac gylcosides and quinones. Of which tannins, polyphenols and saponins were luxuriantly present. Quinones was moderately present. The other compounds, such as flavonoids and cardiac gylcosides were poorly present (Table 1).

Table 1. Presence of primary phytochemicals in A. sessilis leaf extracts

\begin{tabular}{|l|c|c|c|}
\hline \multirow{2}{*}{ Phytochemicals } & \multicolumn{3}{|c|}{ Solvents } \\
\cline { 2 - 4 } & $\begin{array}{c}\text { Petroleum ether } \\
\text { (non-polar) }\end{array}$ & $\begin{array}{c}\text { Acetone } \\
\text { (middle-polar) }\end{array}$ & $\begin{array}{c}\text { Ethanol } \\
\text { (Polar) }\end{array}$ \\
\hline Alkaloids & ++ & -- & -- \\
\hline Terpenoids & ++ & + & -- \\
\hline Flavonoids & + & + & + \\
\hline Tannins & + & ++ & +++ \\
\hline Polyphenols & ++ & +++ & +++ \\
\hline Saponins & -- & ++ & ++ \\
\hline Cardiac gylcosides & -- & +++ & + \\
\hline Quinones & + & + & ++ \\
\hline
\end{tabular}

+, Poor presence; ++, Moderate presence; +++, Luxuriant presence; --, Absence

\subsection{Secondary Phytoconstituents of A. sessilis}

Overall the GC-MS analyses of A. sessilis leaf extracts contains of 17 different secondary compounds, they are depicted in table 2. The petroleum etheric extract of A. sessilis revealed the presence of 7 different secondary compounds \{N-3-Oxopropylcrotonylamide; Dibenz[a,h]anthracene, 5,6,12,13tetrahydro; 6-Formyl-2,2'-bis[1,3-ithiolo[4,5-b][1,4]dithiin-2,2'-diylidene]; 1-phenyl-1-hydro-1(diethoxy-oxo-phosphinyl)-phosphine - (pentacarbonyl) tungsten complex; 2-Pentadecanone, 6,10,14-trimethyl; 9,12-Octadecadienoic acid (Z,Z); 3-Cyanobenzaldehyde dimethyl acetl $\}$. Of which, 2 compounds \{2-Pentadecanone, 6,10,14-trimethyl; 9,12-Octadecadienoic acid (Z,Z) $\}$ are possessed bioactive properties (Tables 2 and 3; Figs. 1 and 1a).

The acetonic extract of $A$. sessilis showed presence of 5 different secondary compounds $\{5-$ Cyclohexyl-1,3,6-trimethyluracil; 2-sec-Butoxy-4,5-dimethylfuran; 19-norpregn-4,18-dien-20-yn-3one; 9,12-Octadecadienoic acid (Z,Z); (13E,16S,17R,18R)-17,18-Epoxy-16,18-dimethyl-10phenyl[11]cytochalasa-6(7),13-dien-1,21-dione\}. Of which, 2 compounds \{5-Cyclohexyl-1,3,6trimethyluracil; 9,12-Octadecadienoic acid (Z,Z) \} are possessed biological properties (Tables 2 and 4; Figs. 2 and $2 a)$.

In the case of ethanolic extract of A. sessilis, 6 different secondary compounds $\{1$-(p-bromophenyl)2,2-dibromocyclopropane; (Z)-1-[(tert-Butyldimethylsilyl)oxy]-6-(phenylthio)-5,7-oc; (2R,3S,4R)- 
Phytochemical Characterization of Alternanthera sessilis and Assessment of its Growth Promoting Potential on the Freshwater Prawn Macrobrachium rosenbergii

1,2:3,4-Di-O-Isopropylidene-1,2,3,4-hexadeca netetraol; 1,7-dideoxy-d-mannoheptulose 1,7bisbenzylamine; 17-Methoxy [2.2.2] (1,3,5) benzene (3,3',3") triphenylmethanophane; Methyl 5trimethylsilyloxy) -bis [O (18) eicosanoate $\}$ were detected. Of which, 2 compounds $\{(2 \mathrm{R}, 3 \mathrm{~S}, 4 \mathrm{R})-$ 1,2:3,4-Di-O-Isopropylidene-1,2,3,4-hexadeca netetraol; 1,7-dideoxy-d-mannoheptulose 1,7bisbenzylamine are possessed bioactive properties (Tables 2 and 5; Fig. 3).

Table 2. Presence of secondary phytochemical compounds in A. sessilis leaf extracts

\begin{tabular}{|c|c|c|c|c|c|c|}
\hline \multicolumn{7}{|c|}{ Solvents } \\
\hline \multicolumn{3}{|c|}{ Petroleum ether (non-polar) } & \multicolumn{2}{|c|}{ Acetone (middle-polar) } & \multicolumn{2}{|c|}{ Ethanol (Polar) } \\
\hline $\begin{array}{c}\text { Peak } \\
\text { RT }\end{array}$ & $\begin{array}{c}\text { Name of the } \\
\text { compounds } \\
\text { identified }\end{array}$ & $\begin{array}{c}\text { Chemical } \\
\text { structure and } \\
\text { molecular } \\
\text { formula }\end{array}$ & $\begin{array}{l}\text { Name of the } \\
\text { compounds } \\
\text { identified }\end{array}$ & $\begin{array}{l}\text { Chemical } \\
\text { structure and } \\
\text { molecular } \\
\text { formula }\end{array}$ & $\begin{array}{l}\text { Name of the } \\
\text { compounds } \\
\text { identified }\end{array}$ & $\begin{array}{l}\text { Chemical } \\
\text { structure } \\
\text { and } \\
\text { molecular } \\
\text { formula }\end{array}$ \\
\hline 5.08 & -- & -- & $\begin{array}{l}\text { 5-Cyclohexyl- } \\
1,3,6- \\
\text { trimethyluracil }\end{array}$ & $\mathrm{C}_{13} \mathrm{H}_{20} \mathrm{~N}_{2} \mathrm{O}_{2}$ & -- & -- \\
\hline 5.53 & $\begin{array}{l}\text { N-3- } \\
\text { Oxopropylcrotony } \\
\text { lamide }\end{array}$ & $\mathrm{C}_{7} \mathrm{H}_{11} \mathrm{NO}_{2}$ & -- & -- & 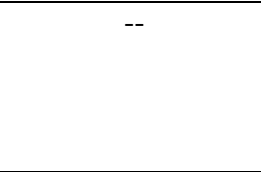 & -- \\
\hline 5.85 & -- & -- & -- & -- & $\begin{array}{l}\text { 1-(p- } \\
\text { bromophenyl)-2,2- } \\
\text { dibromocyclopropa } \\
\text { ne }\end{array}$ & $\mathrm{C}_{9} \mathrm{H}_{7} \mathrm{Br}_{3}$ \\
\hline 8.58 & $\begin{array}{l}\text { Dibenz[a,h]anthra } \\
\text { cene, } 5,6,12,13- \\
\text { tetrahydro }\end{array}$ & & -- & -- & -- & -- \\
\hline 9.57 & -- & -- & -- & -- & $\begin{array}{l}\text { (Z)-1-[(tert- } \\
\text { Butyldimethylsilyl) } \\
\text { oxy]-6- } \\
\text { (phenylthio)-5,7-oc } \\
\text { tadien-4-ol }\end{array}$ & $\mathrm{C}_{20} \mathrm{H}_{32} \mathrm{O}_{2} \mathrm{SS}$ \\
\hline 10.05 & -- & - & $\begin{array}{l}\text { 2-sec-Butoxy-4,5- } \\
\text { dimethylfuran }\end{array}$ & & & \\
\hline 12.09 & $\mathrm{NV}$ & $\mathrm{NV}$ & -- & -- & -- & -- \\
\hline 13.88 & -- & -- & $\mathrm{NV}$ & $\mathrm{NV}$ & -- & -- \\
\hline 14.15 & -- & - & -- & -- & $\begin{array}{l}(2 \mathrm{R}, 3 \mathrm{~S}, 4 \mathrm{R})- \\
1,2: 3,4-\mathrm{Di}-\mathrm{O}- \\
\text { Isopropylidene- } \\
\text { 1,2,3,4-hexadeca } \\
\text { netetraol }\end{array}$ & $\mathrm{C}_{22} \mathrm{H}_{42} \mathrm{O}_{4}$ \\
\hline 16.32 & $\begin{array}{l}\text { 6-Formyl-2,2'- } \\
\text { bis[1,3- } \\
\text { ithiolo[4,5- } \\
\text { b][1,4]dithiin- } \\
\text { 2,2'-diylidene] }\end{array}$ & $\mathrm{C}_{11} \mathrm{H}_{6} \mathrm{OS}_{8}$ & -- & -- & -- & -- \\
\hline 17.85 & -- & -- & $\mathrm{NV}$ & $\mathrm{NV}$ & -- & -- \\
\hline 19.91 & $\begin{array}{l}\text { 1-phenyl-1-hydro- } \\
\text { 1-(diethoxy-oxo- } \\
\text { phosphinyl)- } \\
\text { phosphine - } \\
\text { (pentacarbonyl) } \\
\text { tungsten complex }\end{array}$ & 造 & -- & -- & & \\
\hline 22.29 & -- & -- & $\begin{array}{l}\text { 19-norpregn-4,18- } \\
\text { dien-20-yn-3-one }\end{array}$ & & -- & -- \\
\hline
\end{tabular}


Phytochemical Characterization of Alternanthera sessilis and Assessment of its Growth Promoting Potential on the Freshwater Prawn Macrobrachium rosenbergii

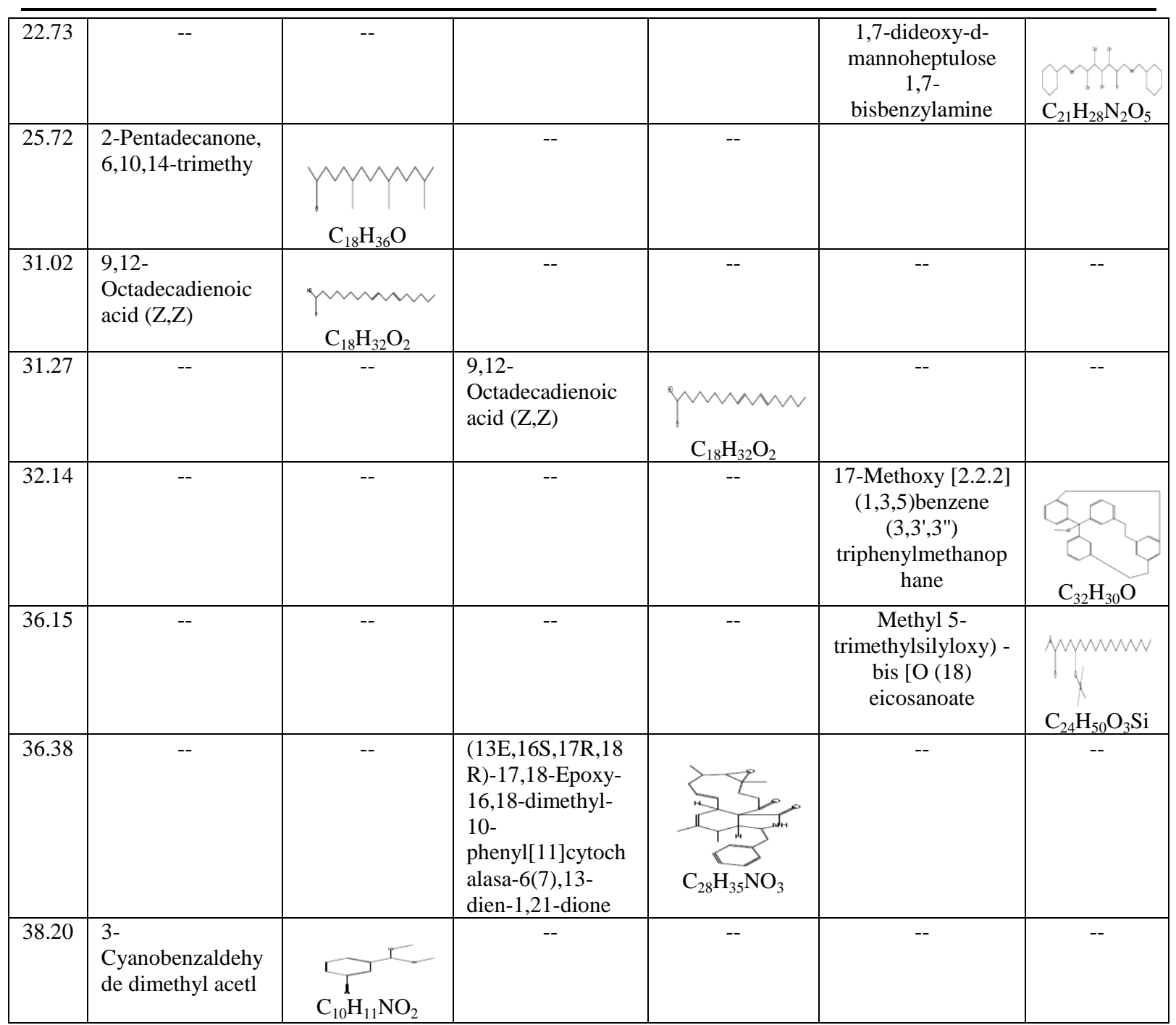

$R T$, Retention time; $N V$, Not validated

Table 3. GC-MS profiles of secondary phytochemical compounds in petroleum etheric extract of A. sessilis leaf

\begin{tabular}{|c|c|c|c|c|c|c|c|c|}
\hline RT & $\begin{array}{l}\text { Name of the } \\
\text { compound }\end{array}$ & $\mathbf{P}$ & MF & MW & $\begin{array}{c}\text { Area } \\
(\%)\end{array}$ & SI & RSI & $\begin{array}{c}\text { Biological properties } \\
\text { by literature only }\end{array}$ \\
\hline 5.53 & N-3-Oxopropylcrotonylamide & 80.59 & $\mathrm{C}_{7} \mathrm{H}_{11} \mathrm{NO}_{2}$ & 141 & 71.58 & 853 & 875 & -- \\
\hline 8.58 & $\begin{array}{l}\text { Dibenz[a,h]anthracene, } \\
\text { 5,6,12,13-tetrahydro }\end{array}$ & 10.08 & $\mathrm{C}_{22} \mathrm{H}_{18}$ & 282 & 0.00 & 365 & 727 & -- \\
\hline 12.09 & NV & NV & NV & NV & NV & $\mathrm{NV}$ & NV & NV \\
\hline 16.32 & $\begin{array}{l}\text { 6-Formyl-2,2'-bis[1,3- } \\
\text { ithiolo[4,5-b][1,4]dithiin-2,2'- } \\
\text { diylidene] }\end{array}$ & 46.22 & $\mathrm{C}_{11} \mathrm{H}_{6} \mathrm{OS}_{8}$ & 410 & 0.00 & 432 & 751 & -- \\
\hline 19.91 & $\begin{array}{l}\text { 1-phenyl-1-hydro-1-(diethoxy- } \\
\text { oxo-phosphinyl)-phosphine - } \\
\text { (pentacarbonyl) tungsten } \\
\text { complex }\end{array}$ & 60.06 & $\mathrm{C}_{15} \mathrm{H}_{16} \mathrm{O}_{8} \mathrm{P}_{2} \mathrm{~W}$ & 570 & 0.02 & 433 & 846 & -- \\
\hline 25.72 & $\begin{array}{l}\text { 2-Pentadecanone, 6,10,14- } \\
\text { trimethyl }\end{array}$ & 60.34 & $\mathrm{C}_{18} \mathrm{H}_{36} \mathrm{O}$ & 268 & 0.02 & 712 & 837 & $\begin{array}{l}\text { Skin creams, lotion, } \\
\text { cosmetic products } \\
\text { (Hussain et el., 2004) }\end{array}$ \\
\hline 31.02 & $\begin{array}{l}\text { 9,12-Octadecadienoic acid } \\
(\mathrm{Z}, \mathrm{Z})\end{array}$ & 29.96 & $\mathrm{C}_{18} \mathrm{H}_{32} \mathrm{O}_{2}$ & 280 & 0.49 & 764 & 792 & $\begin{array}{l}\text { Anti-inflammatory, acne } \\
\text { reductive, and moisture } \\
\text { retentive (Diezel } \text { et al., } \\
\text { 1993; Letawe } \text { et al., 1998; } \\
\text { Darmstadt } \text { et al., 2002) }\end{array}$ \\
\hline 38.20 & $\begin{array}{l}\text { 3-Cyanobenzaldehyde } \\
\text { dimethyl acetl }\end{array}$ & 31.83 & $\mathrm{C}_{10} \mathrm{H}_{11} \mathrm{NO}_{2}$ & 177 & 0.28 & 490 & 837 & -- \\
\hline
\end{tabular}

RT, Retention time; NV, Not validated; P, Probability; MF, Molecular formula; MW, Molecular weight; SI, Similar index; RSI, Reverse similar index 
Phytochemical Characterization of Alternanthera sessilis and Assessment of its Growth Promoting Potential on the Freshwater Prawn Macrobrachium rosenbergii

Table 4. GC-MS profiles of secondary phytochemical compounds in acetonic extract of A. sessilis leaf

\begin{tabular}{|l|l|c|c|c|c|c|c|c|}
\hline RT & $\begin{array}{l}\text { Name of the } \\
\text { compound }\end{array}$ & $\mathbf{P}$ & $\mathbf{M F}$ & $\mathbf{M W}$ & $\begin{array}{c}\text { Area } \\
(\%)\end{array}$ & $\mathbf{S I}$ & $\mathbf{R S I}$ & $\begin{array}{c}\text { Biological properties } \\
\text { by literature only }\end{array}$ \\
\hline 5.08 & $\begin{array}{l}\text { 5-Cyclohexyl-1,3,6- } \\
\text { trimethyluracil }\end{array}$ & 18.28 & $\mathrm{C}_{13} \mathrm{H}_{20} \mathrm{~N}_{2} \mathrm{O}_{2}$ & 236 & 53.96 & 839 & 839 & $\begin{array}{c}\text { Cough suppressant ( De } \\
\text { Blasio } \text { et al., 2012) }\end{array}$ \\
\hline 10.05 & $\begin{array}{l}\text { 2-sec-Butoxy-4,5- } \\
\text { dimethylfuran }\end{array}$ & 5.99 & $\mathrm{C}_{10} \mathrm{H}_{16} \mathrm{O}_{2}$ & 168 & 0.01 & 344 & 799 & -- \\
\hline 13.88 & $\mathrm{NV}$ & $\mathrm{NV}$ & $\mathrm{NV}$ & $\mathrm{NV}$ & $\mathrm{NV}$ & $\mathrm{NV}$ & $\mathrm{NV}$ & $\mathrm{NV}$ \\
\hline 17.85 & $\mathrm{NV}$ & $\mathrm{NV}$ & $\mathrm{NV}$ & $\mathrm{NV}$ & $\mathrm{NV}$ & $\mathrm{NV}$ & $\mathrm{NV}$ & $\mathrm{NV}$ \\
\hline 22.29 & $\begin{array}{l}\text { 19-norpregn-4,18- } \\
\text { dien-20-yn-3-one }\end{array}$ & 4.98 & $\mathrm{C}_{20} \mathrm{H}_{24} \mathrm{O}$ & 280 & 0.03 & 364 & 477 & -- \\
\hline 31.27 & $\begin{array}{l}\text { 9,12-Octadecadienoic } \\
\text { acid (Z,Z) }\end{array}$ & 31.63 & $\mathrm{C}_{18} \mathrm{H}_{32} \mathrm{O}_{2}$ & 280 & 2.75 & 729 & 789 & $\begin{array}{c}\text { Anti-inflammatory, } \\
\text { acne reductive, and } \\
\text { moisture retentive } \\
\text { (Diezel } \text { et al., 1993; } \\
\text { Letawe } \text { et al., 1998; } \\
\text { Darmstadt } \text { et al., 2002) }\end{array}$ \\
\hline 36.38 & $\begin{array}{l}\text { (13E,16S,17R,18R)- } \\
\text { 17,18-Epoxy-16,18- } \\
\text { dimethyl-10- } \\
\text { phenyl[11] } \\
\text { cytochalasa-6(7),13- } \\
\text { dien-1,21-dione }\end{array}$ & 64.26 & $\mathrm{C}_{28} \mathrm{H}_{35} \mathrm{NO}_{3}$ & 433 & 1.03 & 390 & 896 & -- \\
\hline
\end{tabular}

RT, Retention time; NV, Not validated; P, Probability; MF, Molecular formula; MW, Molecular weight; SI, Similar index; RSI, Reverse similar index

Table 5. GC-MS profiles of secondary phytochemical compounds of A. sessilis extracted with ethanol

\begin{tabular}{|c|l|c|c|c|c|c|c|c|}
\hline RT & $\begin{array}{l}\text { Name of the } \\
\text { compound }\end{array}$ & $\mathbf{P}$ & $\mathbf{M F}$ & $\mathbf{M W}$ & $\begin{array}{c}\text { Area } \\
(\%)\end{array}$ & $\mathbf{S I}$ & $\mathbf{R S I}$ & $\begin{array}{c}\text { Biological } \\
\text { properties } \\
\text { by literature only }\end{array}$ \\
\hline 5.85 & $\begin{array}{l}\text { 1-(p-bromophenyl)-2,2- } \\
\text { dibromocyclopropane }\end{array}$ & 7.17 & $\mathrm{C}_{9} \mathrm{H}_{7} \mathrm{Br}_{3}$ & 352 & 38.87 & 288 & 519 & -- \\
\hline 9.57 & $\begin{array}{l}\text { (Z)-1-[(tert- } \\
\text { Butyldimethylsilyl)oxy]- } \\
\text { 6-(phenylthio)-5,7-oc } \\
\text { tadien-4-ol }\end{array}$ & 16.10 & $\mathrm{C}_{20} \mathrm{H}_{32} \mathrm{O}_{2} \mathrm{SSi}$ & 364 & 0.46 & 395 & 662 & -- \\
\hline 14.15 & $\begin{array}{l}\text { (2R,3S,4R)-1,2:3,4-Di-O- } \\
\text { Isopropylidene-1,2,3,4- } \\
\text { hexadeca netetraol }\end{array}$ & 42.30 & $\mathrm{C}_{22} \mathrm{H}_{42} \mathrm{O}_{4}$ & 370 & 0.53 & 459 & 824 & $\begin{array}{l}\text { Detergents and } \\
\text { cleaning agents } \\
\text { (OECD, (1994) }\end{array}$ \\
\hline 22.73 & $\begin{array}{l}\text { 1,7-dideoxy-d- } \\
\text { mannoheptulose 1,7- } \\
\text { bisbenzylamine }\end{array}$ & 9.02 & $\mathrm{C}_{21} \mathrm{H}_{28} \mathrm{~N}_{2} \mathrm{O}_{5}$ & 388 & 1.73 & 400 & 497 & $\begin{array}{c}\text { Allergy, hay fever, } \\
\text { cold (WebMD, } \\
2016)\end{array}$ \\
\hline 32.14 & $\begin{array}{l}\text { 17-Methoxy [2.2.2] } \\
\text { (1,3,5)benzene (3,3',3") } \\
\text { triphenylmethanophane }\end{array}$ & 91.90 & $\mathrm{C}_{32} \mathrm{H}_{30} \mathrm{O}$ & 430 & 14.36 & 558 & 670 & - \\
\hline 36.15 & $\begin{array}{l}\text { Methyl 5- } \\
\text { trimethylsilyloxy) -bis [O } \\
\text { (18) eicosanoate }\end{array}$ & 7.55 & $\mathrm{C}_{24} \mathrm{H}_{50} \mathrm{O}_{3} \mathrm{Si}$ & 414 & 5.09 & 292 & 546 & \\
\hline
\end{tabular}

RT, Retention time; NV, Not validated; P, Probability; MF, Molecular formula; MW, Molecular weight; SI, Similar index; RSI, Reverse similar index 
Phytochemical Characterization of Alternanthera sessilis and Assessment of its Growth Promoting Potential on the Freshwater Prawn Macrobrachium rosenbergii

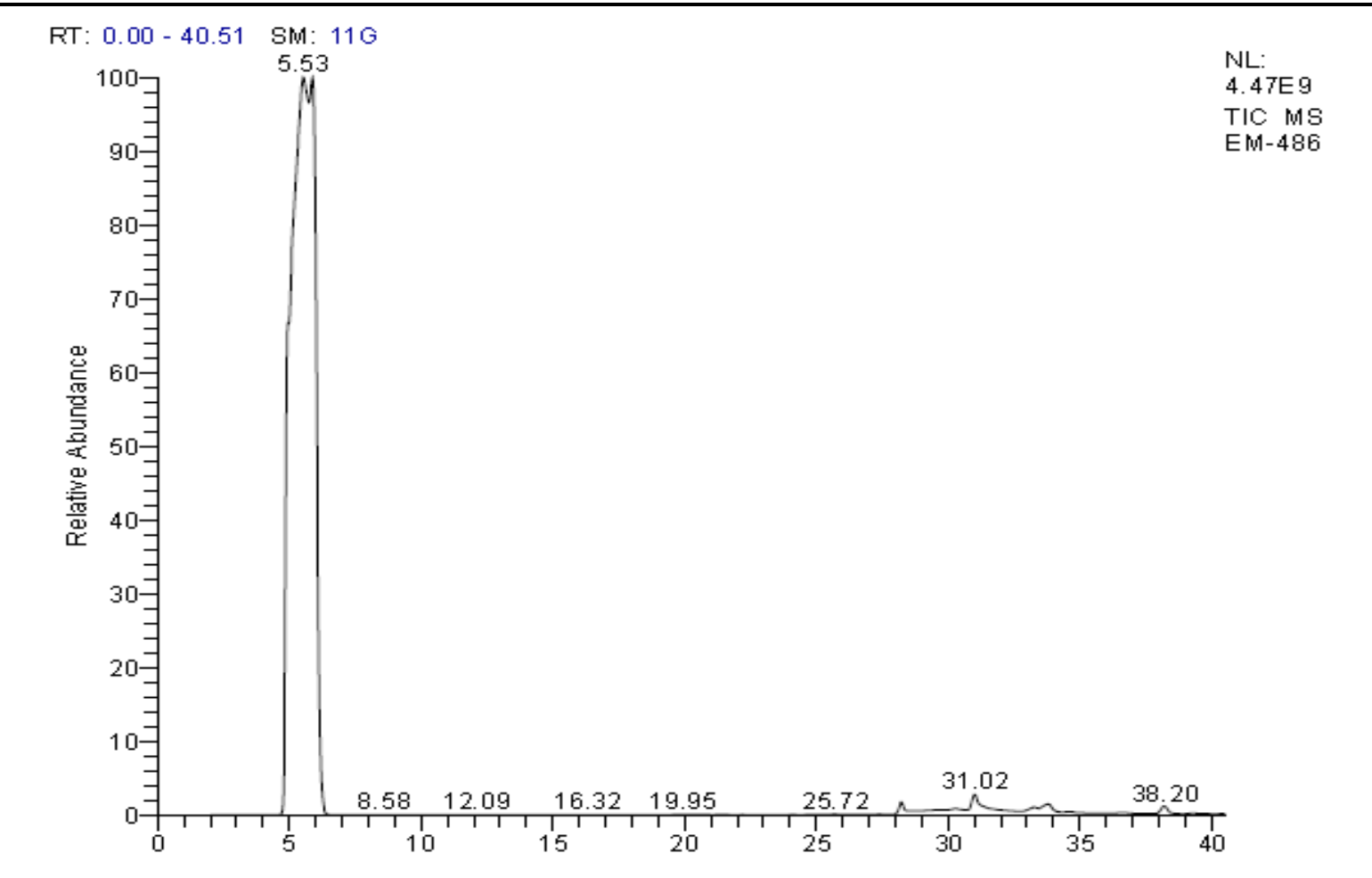

Fig. 1. GC-MS chromatogram of petroleum etheric extracted A. sessilis (Relative abundance up to 100)

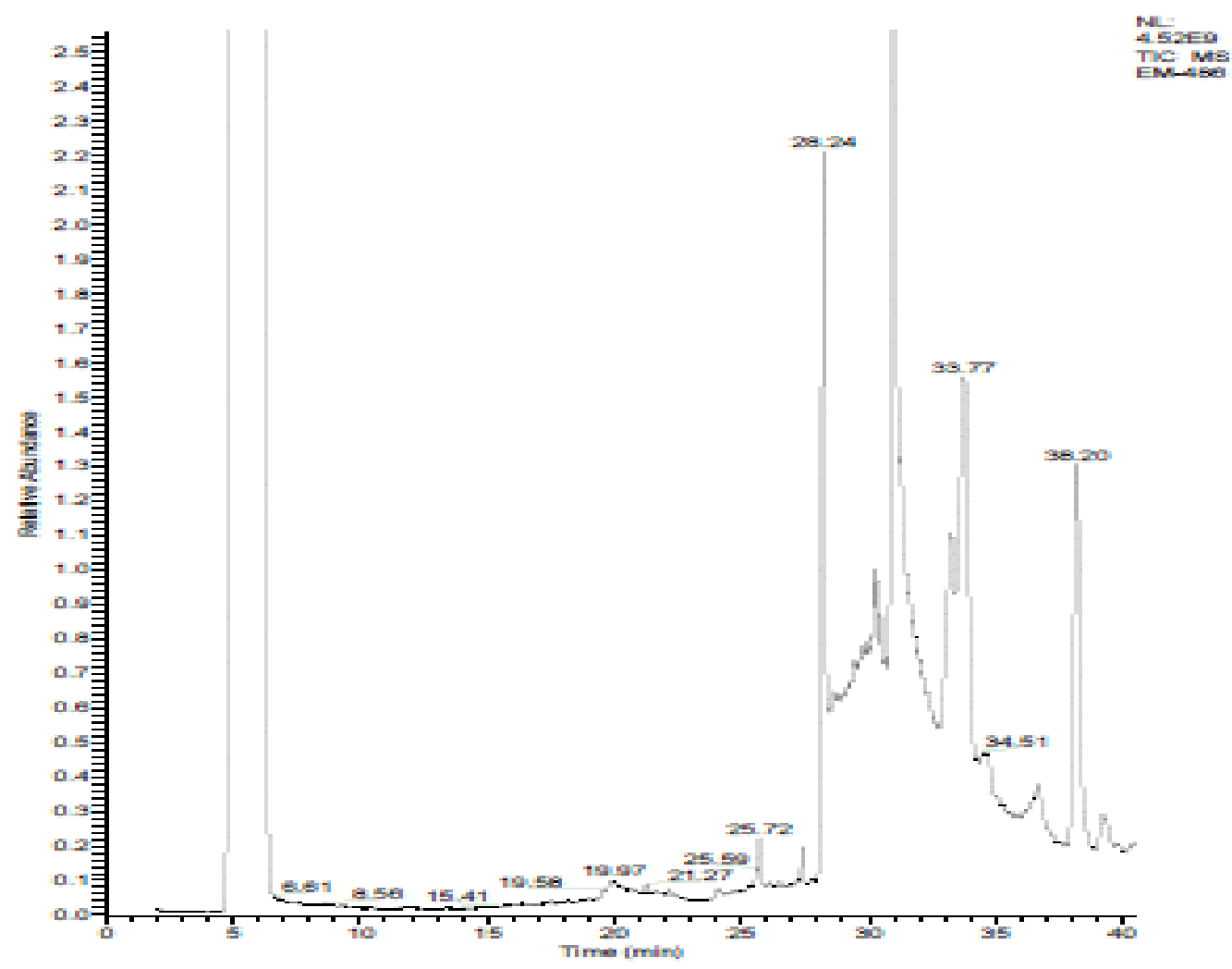

Fig. 1a. GC-MS chromatogram of petroleum etheric extracted A. sessilis (Relative abundance up to 2.5) 
Phytochemical Characterization of Alternanthera sessilis and Assessment of its Growth Promoting Potential on the Freshwater Prawn Macrobrachium rosenbergii

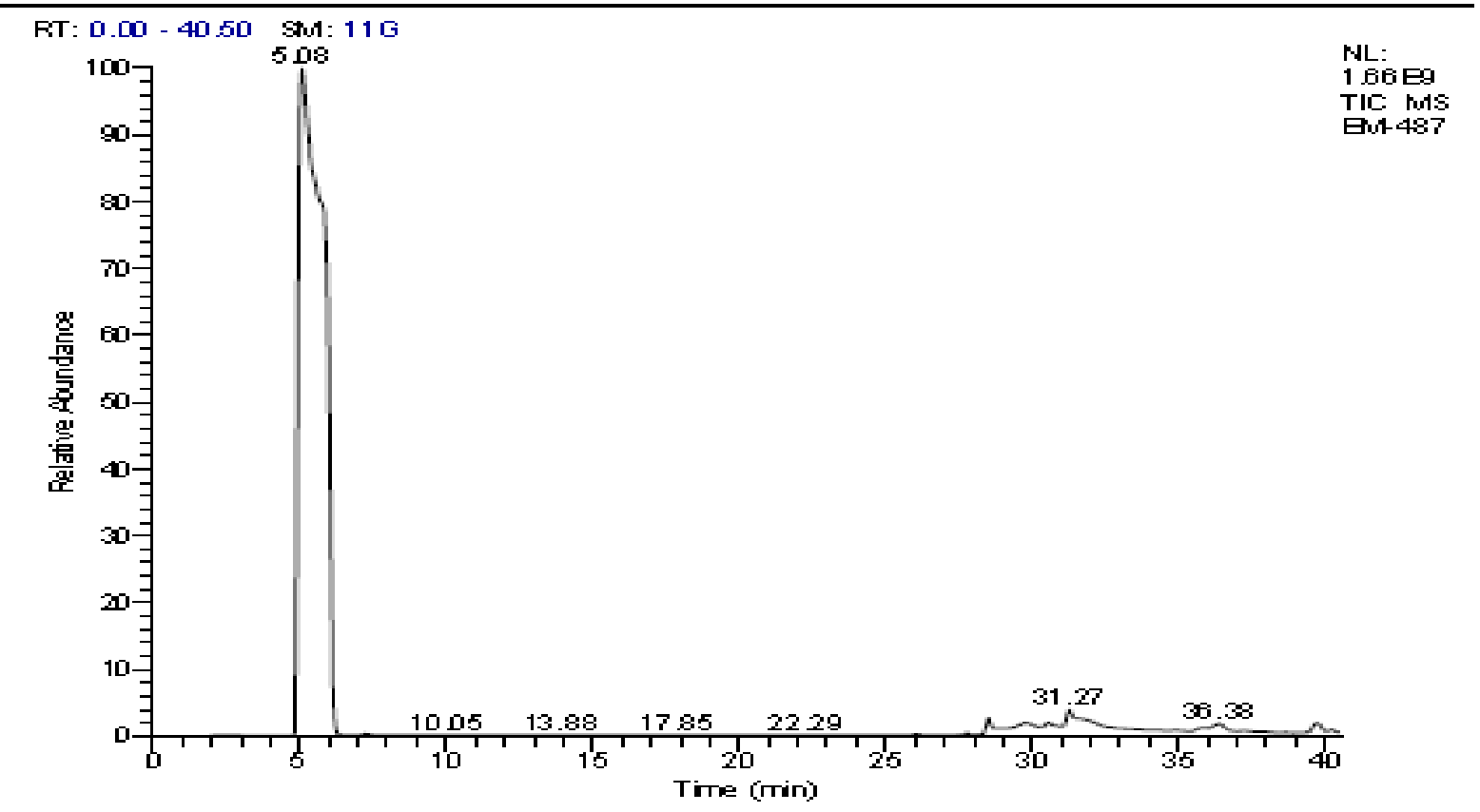

Fig. 2. GC-MS peak level in the chromatogram graph of acetonic extract of A. sessilis (Relative abundance up to 100)

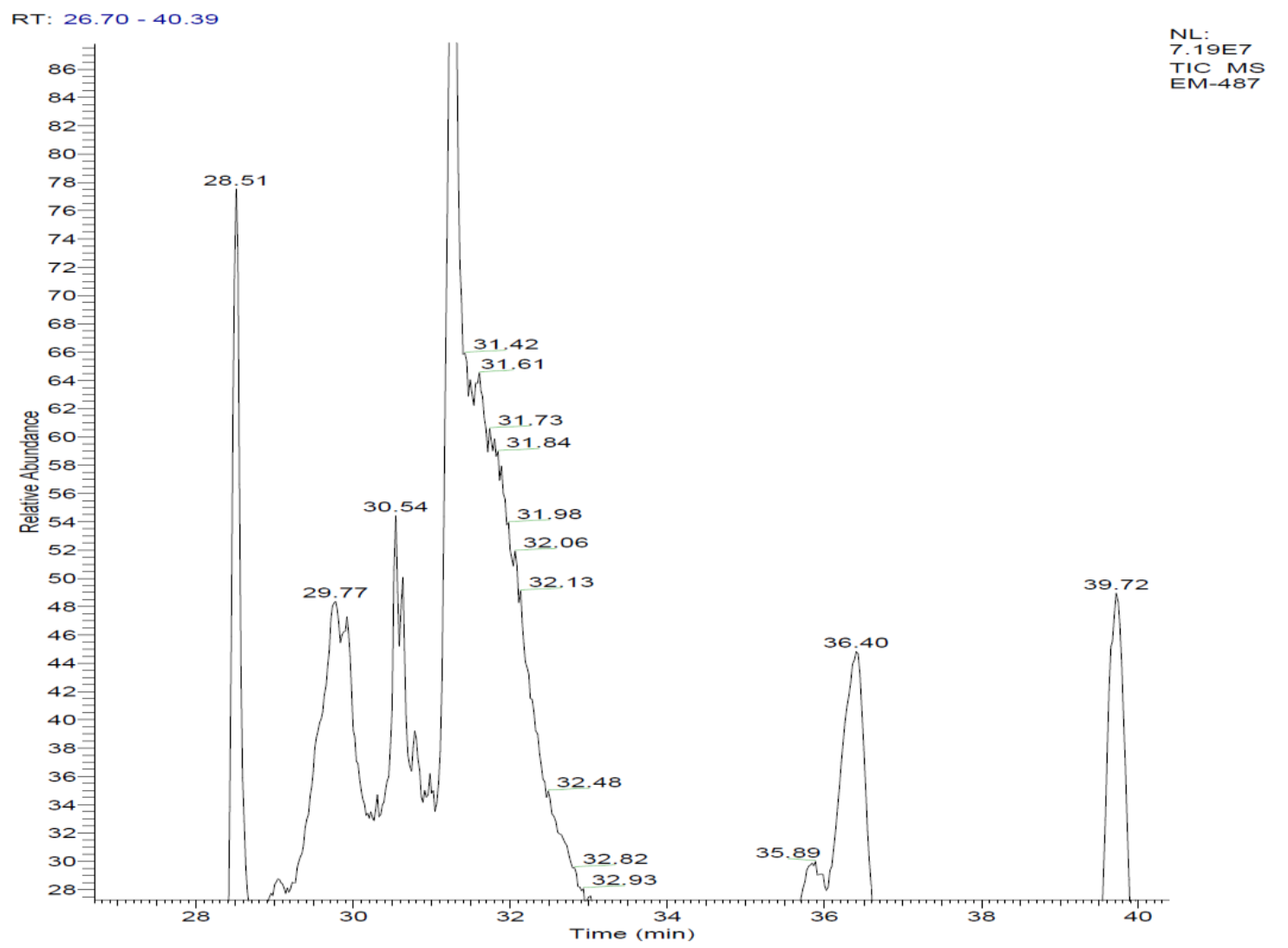

Fig. 2a. GC-MS peak level in the magnified chromatogram graph of acetonic extract of A. sessilis (Relative abundance up to 56)

\subsection{Nutritional Indices}

The morphometric analyses revealed that the nutritional indices such as SR, WG, SGR and PER were found to be significantly increased in $1 \%$ ethanolic extract incorporated feed fed prawns $(p<0.05)$ followed by $0.5 \%$ and $0.1 \%$ when compared with control (Table 6). In the case of FCR, the data naturally appeared in the decreasing trend that the lowest FCR was recorded in $1 \%$ of ethanolic extract incorporated feed fed prawns, which reflects the best quality of feed. 
Phytochemical Characterization of Alternanthera sessilis and Assessment of its Growth Promoting Potential on the Freshwater Prawn Macrobrachium rosenbergii

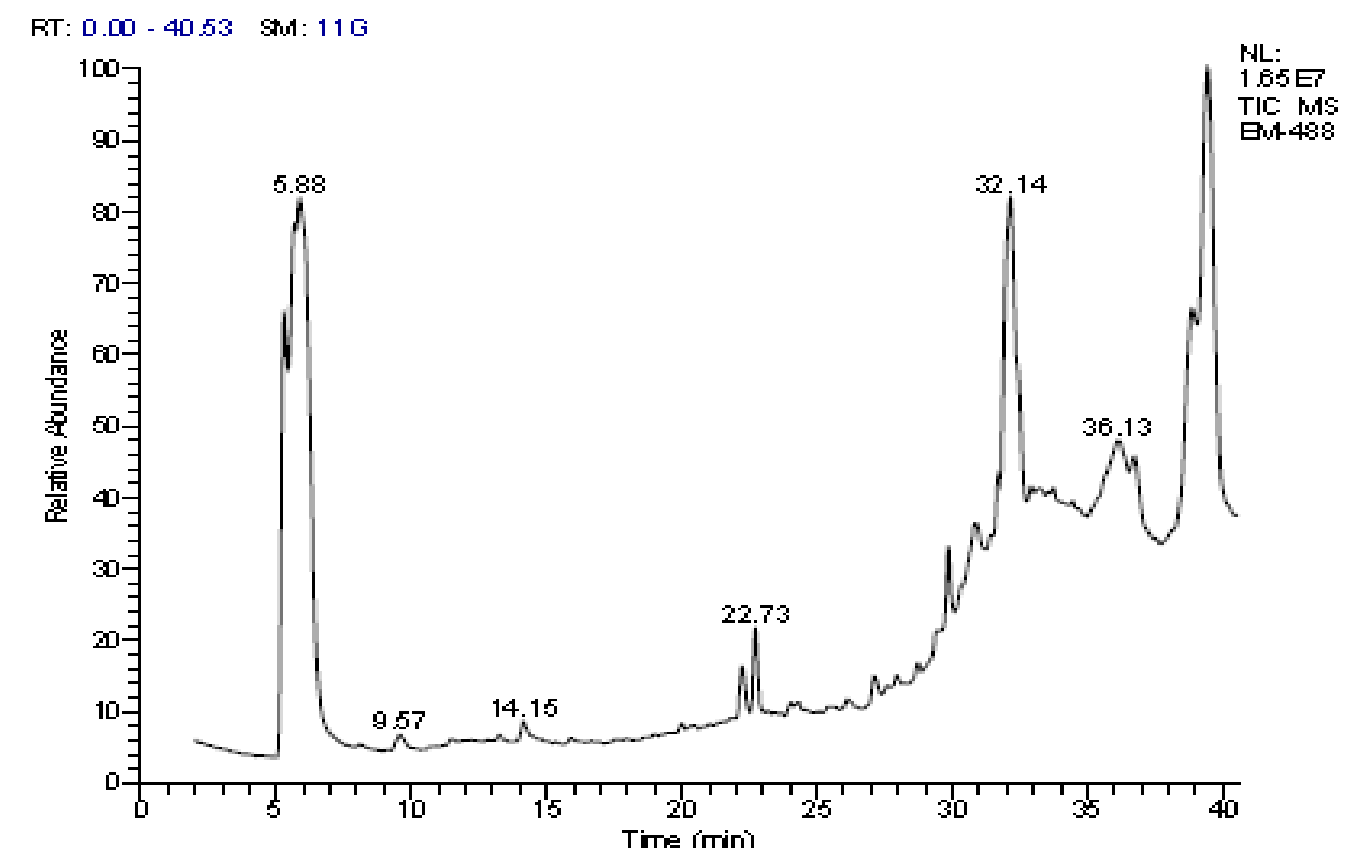

Fig. 3. GC-MS peak level in the chromatogram graph of ethanolic extract of A. sessilis (Relative abundance up to 100$)$

Table 6. Nutritional indices of $M$. rosenbergii fed with ethanolic extract of A. sessilis leaf incorporated feeds

\begin{tabular}{|l|c|c|c|c|}
\hline \multirow{2}{*}{ Parameter } & Control & \multicolumn{3}{|c|}{ Ethanolic extract } \\
\cline { 2 - 5 } & BI & BI+0.1\% & BI+0.5\% & BI+1\% \\
\hline SR (\%) & $66.66 \pm 6.66^{\mathrm{d}}$ & $81.11 \pm 5.09^{\mathrm{c}}$ & $85.55 \pm 6.33^{\mathrm{b}}$ & $88.66 \pm 3.33^{\mathrm{a}}$ \\
\hline Length (cm) & $4.09 \pm 0.22^{\mathrm{d}}$ & $5.15 \pm 0.18^{\mathrm{bc}}$ & $5.25 \pm 0.15^{\mathrm{b}}$ & $5.40 \pm 0.18^{\mathrm{a}}$ \\
\hline Weight (g) & $0.59 \pm 0.06^{\mathrm{d}}$ & $1.14 \pm 0.14^{\mathrm{bc}}$ & $1.20 \pm 0.21^{\mathrm{b}}$ & $1.41 \pm 0.22^{\mathrm{a}}$ \\
\hline WG (g) & $0.44 \pm 0.01^{\mathrm{d}}$ & $0.99 \pm 0.11^{\mathrm{bc}}$ & $1.05 \pm 0.23^{\mathrm{b}}$ & $1.25 \pm 0.24^{\mathrm{a}}$ \\
\hline SGR (\%) & $0.68 \pm 0.02^{\mathrm{d}}$ & $0.96 \pm 0.02^{\mathrm{bc}}$ & $0.98 \pm 0.13^{\mathrm{b}}$ & $1.09 \pm 0.09^{\mathrm{a}}$ \\
\hline FCR (g) & $3.05 \pm 0.12^{\mathrm{a}}$ & $1.63 \pm 0.10^{\mathrm{b}}$ & $1.53 \pm 0.05^{\mathrm{bc}}$ & $1.29 \pm 0.09^{\mathrm{d}}$ \\
\hline PER (g) & $0.77 \pm 0.03^{\mathrm{d}}$ & $1.89 \pm 0.07^{\mathrm{c}}$ & $2.07 \pm 0.05^{\mathrm{b}}$ & $2.24 \pm 0.11^{\mathrm{a}}$ \\
\hline
\end{tabular}

Each value is mean \pm standard deviation of three individual observations.

Initial length and weight were $2.63 \pm 0.11 \mathrm{~cm}$ and $0.15 \pm 0.02$ respectively

Mean values within the same row sharing different alphabetical letter superscripts are statistically significant at $p<0.05$ (one-way ANOVA and subsequent post hoc multiple comparison with DMRT.

BI, basal ingredients; SR, survival rate; WG, weight gain, SGR, specific growth rate; FCR, food conversion ratio; PER, protein efficiency ratio

\subsection{Biochemical constituents}

The basic biochemical constituents, such as total protein, total carbohydrate, total lipid and ash were found to be significantly increased in $1 \%$ ethanolic extract incorporated feed fed prawns $(p<0.05)$ followed by $0.5 \%$ and $0.1 \%$ when compared with control (Table 7). In the case of moisture content, the most decrease was found to be in $1 \%$ ethanolic extract incorporated feed fed prawns $(p<0.05)$ followed by $0.5 \%$ and $0.1 \%$ when compared with control (Table 7).

Table 7. Concentration of biochemical constituents (mg/g wet wt.) in M. rosenbergii fed with ethanolic extract of $A$. sessilis leaf incorporated feeds

\begin{tabular}{|l|c|c|c|c|c|}
\hline \multirow{2}{*}{ Parameters } & \multirow{2}{*}{ Initial } & Control & \multicolumn{3}{c|}{ Ethanolic extract } \\
\cline { 3 - 6 } & & BI & BI+0.1\% & BI+0.5\% & BI+1\% \\
\hline Total protein & $43.91 \pm 2.74$ & $82.78 \pm 4.47^{\mathrm{d}}$ & $115.93 \pm 3.44^{\mathrm{c}}$ & $120.74 \pm 4.57^{\mathrm{b}}$ & $133.5 \pm 3.77^{\mathrm{a}}$ \\
\hline Total carbohydrate & $21.06 \pm 1.98$ & $30.35 \pm 2.59^{\mathrm{d}}$ & $45.69 \pm 3.13^{\mathrm{bc}}$ & $46.94 \pm 2.27^{\mathrm{b}}$ & $49.01 \pm 2.51^{\mathrm{a}}$ \\
\hline Total lipid & $9.32 \pm 2.05$ & $18.21 \pm 2.54^{\mathrm{d}}$ & $25.89 \pm 2.54^{\mathrm{bc}}$ & $26.91 \pm 2.04^{\mathrm{ab}}$ & $27.94 \pm 1.65^{\mathrm{a}}$ \\
\hline Moisture (\%) & $76.66 \pm 4.32$ & $65.33 \pm 2.08^{\mathrm{a}}$ & $53.33 \pm 3.64^{\mathrm{b}}$ & $52.66 \pm 2.45^{\mathrm{bc}}$ & $50.33 \pm 2.58^{\mathrm{d}}$ \\
\hline Ash (\%) & $10.65 \pm 1.02$ & $13.18 \pm 1.95^{\mathrm{c}}$ & $17.91 \pm 1.57^{\mathrm{bc}}$ & $18.72 \pm 1.83^{\mathrm{ab}}$ & $19.24 \pm 1.31^{\mathrm{a}}$ \\
\hline
\end{tabular}

Each value is mean \pm standard deviation of three individual observations. 
Phytochemical Characterization of Alternanthera sessilis and Assessment of its Growth Promoting Potential on the Freshwater Prawn Macrobrachium rosenbergii

Mean values within the same row sharing different alphabetical letter superscripts are statistically significant at $p<0.05$ (one-way ANOVA and subsequent post hoc multiple comparison with DMRT

\section{BI, basal ingredients}

\section{DisCussion}

Similar to that of the present study, the presence of alkaloids, flavonids, phenols, steroids, terpenoids, saponins and glycoside have been reported in ethanolic extract of A. sessilis (Sivakumar and Sunmathi, 2016). The presence of other phyto-constituents such as proteins, amino acids, carbohydrates, fixed oil and sterols have also been reported in A. sessilis by several workers (Debnath et al., 2014; Sivakumar and Sunmathi, 2016). The phytochemicals, such as flavonoids, anthrax quinines and terpenes stimulate glucose uptake in cells (Latha and Pari, 2003). Certain flavonoids exhibited hypoglycemic activity (Ahmad et al., 2000) and also beta cell regeneration in pancreas (Latha and Pari, 2003). The primary phytochemicals serve as health tonic in aquaculture nutrition (Citarasu, 2010; Pourmoghim et al., 2015).

The detected hexadecanoic acid, octadecanoic acid and propanoic acid in the present study have also been reported to be present in the hydro-alcoholic extraction of A. sessilis (Shabi et al., 2010).

The detected hypocholesterolemic compounds such as 9, 12-Octadecadienoic acid (Z,Z)- and Octadecanoic acid have also been reported in Justicia wynaadensis (Ponnamma and Manjunath, 2012). The other compounds reported in J. wynaadensis are gamma-Tocopherol, Vitamin E, Ergost5- en-3.Beta.-ol and Stigmasta-5, 22-dien-3.beta.-ol (Ponnamma and Manjunath, 2012).

Vinodh and Senthil Kumar (2014) have reported that the aqueous extract of A. sessilis leaf contains the following secondary phytochemicals: 1-vinylclohexa-1,4-diene; 1-isopropylcyclohexa-1,4dienein; 5-butyl-4-methylnaphalen-1(4H)-one; 4a,8-dimethyl-1-methylene-1,4,4a,4b,5,8,8a,9,10,10 adecahydrophenanthrene; 1-(1-hydroxyethyl)-1-(methoxymethyl)-7a-methyloctahydro-3Hcyclopropa[a]naphthalene-2(7bH)-one; 10,13,17- trimethyl-3-methyylene 2,3,4,5,6,10,12,13,14,15, 16,17-dodecahydro-1Hcyclopenta [a] phenanthrene; 1,1-bis(1-methoxyvinyl)-9b-methyl-4,5,6,7,8,9, 9a,9b,9cdecahydro-1H-cyclopropa [c]phenanthrene; 1,1-bis(1-methoxyvinyl)-9b-methyl-2methylenetetradecahydro-1H-cyclopropa [c] phenanthrene; Dimethyl 17-ethyl-9b-methyl-2-oxo-1a,2dihydro-7 Hcyclopropa [c] phenanthrene-1,1 $(9 \mathrm{aH}, 9 \mathrm{bH}, 9 \mathrm{cH})$ - dicarboxylate and 7-ethyl-1,1-bis(1methoxyvinyl)-2,7,9b-trimethyl-1a,2,7,8,9a,9b,9c-octahydro-1Hcyclopropa [c] phenanthrene. None of the compound was detected in the present study.

The phytocomponents derived from various plants have been used in traditional medicine for the treatment of several diseases. Recent studies showed that the incorporation of medicinal plants (raw material, extracts and phytocomponents) as a source of fish/prawn feed, which stimulate the growth and immune system (Chakrabarti et al., 2012; Bhavan et al., 2014a,b; Pourmoghim et al., 2015; Dhanalakshmi et al., 2016).

Similar to that of the elevated SR, WG, SGR and PER, and elevated total protein, carbohydrate and lipid recorded in the present study in ethanolic extract of $A$. sessilis incorporated feed fed prawns, it has also been reported in Nutripro-aqua, herbal based diet fed M. rosenbergii (Kesavanth et al., 2003). The increased nutritional indices has also been reported in freshwater prawns fed with Alteranthera sessilis, Eclipta alba, Cissus quadrangularis, Allium sativum, Andrographis paniculata, Coriandrum sativum, Curcuma longa, Menthe arvensis, Murraya koenigii, Ocimium sanctum, Trigonella foenumgraecum, Withania somnifera, Zingiber officinale, Cynodon doctylon, Syzygium cumini, Phylanthus emblica, Azadirachta indica, Ricinus communis, Papaver somniferum, Elettaria cardamomum, Foeniculum vulgare, Syzygium aromaticum, Mentha arvensis, and Trigonella foenum-graecum incorporated feeds (Bhavan et al., 2011; Bhavan et al., 2012; Shanthi et al., 2012; Poongodi et al., 2012; Radhakrishanan et al., 2013; Bhavan et al., 2013a,b; Bhavan et al., 2014a,b; Dhanalakshmi et al., 2016).

Generally herbs are good appetizer, they contain bioactive compounds which stimulate the feeding rate and food consumption ratio, and thus improved biochemical constituents in prawns have been reported (Sambhu and Jayaprakash, 2001; Bhavan et al., 2012, 2013a,b; 2014a,b). Herbal growth promoters helped to induce the transcription, leading to increased RNA, which coupled with increased amino acid and finally enhanced protein synthesis in Penaeus monodon (Citarasu, 2010). It has been 
Phytochemical Characterization of Alternanthera sessilis and Assessment of its Growth Promoting Potential on the Freshwater Prawn Macrobrachium rosenbergii

reported that, $W$. somnifera supplemented spawners showed higher protein values in the haemolymph as well as positively regulated the larval quality in P. monodon (Babu et al., 2008).

\section{CONCLUSION}

In the present study, the primary phytochemicals and secondary bioactive compounds present in ethanolic extract of $A$. sessilis have the potency to enhance the growth, survival and nutritional quality of $M$. rosenbergii. Hence, A. sessilis leaf can be taken as a feed additive in on-farming feed preparation, and thus, sustainable aquaculture of freshwater prawns can be promoted.

\section{ACKNOWLEDGEMENT}

The authors are gratefully acknowledged the South India Textile Research Association (SITRA), Coimbatore, Tamil Nadu, India, for providing GC-MS outsourcing service. Animal Feed Analytical and Quality Assurance Laboratory (AFAQAL), Veterinary College and Research Institute, TANUVAS, Namakkal, Tamil Nadu, India, is acknowledged for proving the outsourcing service for analysis of feed proximate composition.

\section{REFERENCES}

Aarumugam, P, Bhavan, P.S., Muralisankar, T., Manickam, N., Srinivasan, V., and Radhakrishnan, S. (2013). Growth of Macrobrachium rosenbergii fed with mango seed kernel, banana peel and papaya peel incorporated feeds. Int. J. App. Biol. Pharm. Tech., 4(2): 12-25.

Ahmad, M., Akhtar, M.S., Malik, T., and Gilani, A.H. (2000). Hypoglycemic action of flavonoids fraction of Cuminum nigrum seeds. Phytotherapy Res. 14: 103-106.

AOAC (1995). Official methods of analysis of AOAC international. 2 vols. 16th edn. Arlington, VA, USA, Association of Analytical Communities.

APHA (2005). Standard Methods for the Examination of Water and Wastewater, 19th Edn, American Public Health Association, New York.

Babu, M.M., Sivaram, V., Immanuel, G., Citarasu, T., and Punitha, S.M.J. (2008). Effects of herbal enriched Artemia supplementation over the reproductive performance and larval quality in spent spawners of the tiger shrimp (Penaeus monodon). Turk. J. Fish. Aqua. Sci., 8: 301-307.

Barnes, H., and Blackstock, J. (1973). Estimation of lipids in marine animals and tissues. Detail investigation of the sulpho-phosphovanillin method for total lipids. J. Exp. Mar. Biol. Ecol., 12:103-118.

Bhavan, P.S., and Radhakrishnan, S. (2012). Nutritional indices and biochemical constituents in the prawn Macrobrachium malcolmsonii fed with formulated feeds. Int. J. Pharma. Bio Sci., 3(2): 327-336.

Bhavan, P.S., Anisha, T.C., Srinivasan, V., Muralisankar, T., and Manickam, N. (2014a). Effects of spices, Papaver somniferum, Elettaria cardamomum, Foeniculum vulgare and Syzygium aromaticum on growth promotion in Macrobrachium malcolmsonii early juveniles. Int. J. Pure. App. Biosci., 2(6): 120-131.

Bhavan, P.S., Jayanthi, S., and Rabecca, A.A. (2011). Growth performance of the freshwater prawn Macrobrachium rosenbergii post larvae fed with Ocimun sanctum (Tulsi) and Withania somnifera (Ashwagandha) incorporated feeds. Int. J. Biol. Res. Develop., 1: 34-53.

Bhavan, P.S., Manickam, N., and Radhakrishnan, S. (2012). Influence of herbal greens, Murraya koenigii, Coriandrum sativum and Menthe arvensis on growth performance of the freshwater prawn Macrobrachium rosenbergii post larvae. Res. J. Biotech., 7: 149-157.

Bhavan, P.S., Mohammedsiddiq, S., Srinivasan, V., Muralisankar, T., and Manickam, N. (2014b). Effects of seeds of medicinal plants, Syzygium cumini, Phylanthus emblica, Azadirachta indica and Ricinus communis on growth promotion in Macrobrachium malcolmsonii early juveniles. Int. J. Res. Stud. Biosci., 2 (11): 95-106.

Bhavan, P.S., Nandhini Devi, N., Muralisankar, T., Manickam, N., Radhakrishnan, S., and Srinivasan, V. (2013b). Effect of Myristica fragrans, Glycyrrhiza glabra and Quercus infectoria on growth promotion in the prawn Macrobrachium rosenbergii. Int. J. Life Sci. Biotech. Pharma Res., 2: 169-182.

Bhavan, P.S., Radhakrishnan, S., Seenivasan, C., Shanthi, R., Poongadi, R., and Kannan, S. 2010. Proximate composition and profiles of amino acid and fatty acids in the muscle of adult males and females of commercially viable prawn species Macrobrachium rosenbergii collected from natural environments. Int. J. Biol., 2, 107-119.

Bhavan, P.S., Saranya, C., Manickam, N., Muralisankar, T., Radhakrishnan, S., and Srinivasan, V. (2013a). Effects of Piper longum, Piper nigram and Zingiber officinale on survival, growth, activities of digestive enzymes and contents of total protein, vitamins and minerals in the freshwater prawn Macrobrachium rosenbergii. Elix. Bio Tech., 58: 14824-14828. 
Phytochemical Characterization of Alternanthera sessilis and Assessment of its Growth Promoting Potential on the Freshwater Prawn Macrobrachium rosenbergii

Castell, J.D., and Tiews, K. (1980). Report on the EIFAC, IUNS and ICES working group on the standardization of methodology in fish nutrition research. Hamburg, Federal Republic of Germany, EIFAC Technical Paper.

Chakrabarti, R., and Rao, Y.V. (2012). Achyranthes aspera enhances immunity and antigen clearance in common carp, Cyprinus carpio L. J Fish Dis., 35: 389-392.

Chandrasekaran, V.S., Sharma, P. (1997). Biology and culture of freshwater prawns in North India. Fish. Chim., 17: 7-9.

Citarasu, T. (2010). Herbal biomedicines: a new opportunity for aquaculture industry. Aqua. Int. 18: 403-414. doi: 10.1007/s10499-009-9253-7.

D’Abramo, L.R., Sheen, S.S. (1994). Nutritional requirements, feed formulation and feeding practices for intensive culture of the freshwater prawn Macrobrachium rosenbergii. Rev. Fish. Sci., 2: 1-21.

Darmstadt, G.L., Mao-Qiang, M., Chi, E., Saha, S.K., Ziboh, V.A., Black, R.E., Santosham, M., and Elias, P.M. (2002). Impact of topical oils on the skin barrier: possible implications for neonatal health in developing countries. Acta Paed., 91(5): 546-554.

De Blasio, F., Dicpinigaitis, P.V., Rubin, B.K., De Danieli, G., Lanata, L., and Zanasi, A. (2012). An observational study on cough in children: Epidemiology, impact on quality of sleep and treatment outcome. Cough. 8 (1): 1. https://en.wikipedia.org/wiki/Levodropropizine.

Debnath, M., Nandi, M., and Biswas, M. (2014). A critical pharmacognostic evaluation and preliminary phytochemical investigation of Alternanthera sessilis (L.) R. BR. Leaves. Ind. J. Pharma. Sci. Res., 4(2): 71-74.

Dhanalakshmi, K., Bhavan, P.S, Rajkumar, G., Nathiya, V., Srinivasan, V., and Satgurunathan, T. (2016) Phytochemical Characterization of Couch Grass (Cynodon dactylon) and Its Growth PromotingPotential on the Freshwater Prawn Macrobrachium rosenbergii Post-Larvae. Brit. Biotech. J., 14(2): 1-24.

Diezel, W.E., Schulz, E., Skanks, M., and Heise, H. (1993). Plant oils: Topical application and antiinflammatory effects (croton oil test). Dermatol. Monat., 179: 173.

Folch, J., Lees, M., and Bloane-Stanley, G.H. (1957). A simple method for the isolation and purification of total lipids from animal tissues. J. Biol. Chem., 266: 497-509.

Guerra, R.N.M., Pereira, H.A.W., Silveira, L.M.S., and Olea, R.S.G. (2003) Immunomodulatory properties of Alternanthera tenella Colla aqueous extracts in mice. Braz. J. Med. Biol. Res., 36: 1215- 1219.

Gupta, A.K. (2014). Alternanthera sessilis. The IUCN Red List of Threatened Species. Version 2014. http://www.iucnredlist.org.

Hilou, A., Nacoulmaa, O.G., Guiguemdeb, T.R. (2006). In vivo antimalarial activities of extracts from Amaranthus spinosus L. and Boerhaavia erecta L. in mice. J. Ethno pharma., 103, 236-240.

Hussaina, A., Arnoldb, J.J., Khana, M.A., and Ahsan, F. (2014). Absorption enhancers in pulmonary protein delivery. J. Contr. Rel., 94(1): 15-24.

Kesavanth, P., Gangadhara, B, and Khadri, S. (2003). Growth enhancement of carp and prawn through dietary sodium chloride supplementation. Aquacult. Asia, 8: 4.

Langer, S., Bakhtiyar, Y., and Lakhnotra, R. (2011). Replacement of fishmeal with locally available ingredients in diet composition of Macrobrachium dayanum. Afri. J. Agri. Res. 6(5): 1080-1084.

Latha, M., Pari, L. (2003). Anti hyperglycemic effect of Cassia auriculata in experimental diabetes and its effect on key metabolic enzymes in carbohydrate metabolism. Clin. Exp. Pharm. Physiol., 30: 38-43.

Letawe, C., Boone, M., and Pierard, G.E. (1998). Digital image analysis of the effect of topically applied linoleic acid on acne microcomedones. Clin. Exp. Dermatol., 23(2): 56-58.

Lowry, O.H., Rosenbrough, W.J., Fair, A.L., and Randall, R.J. (1951). Protein measurement with the Folin phenol reagent. J. Biol. Chem., 193: 265-275.

Mariappan, P., Balamurugan, P., and Balasundaram C. (2003). Freshwater prawn Macrobrachium nobili a promising candidate for rural nutrition. Curr. Sci., 8: 13-14.

Mitra, G., Chattopadhyay, D.N., and Mukhopadhyay, P.K. (2005). Nutrition and feeding in freshwater prawn (Macrobrachium rosenbergii) farming. Aqua feeds: Formulation and Beyond, 2: 17-19.

OECD. (1994). Screening Information Data Set (SIDS) of OECD high production volume chemicals programme, UNEP publications. ttp://www.inchem.org/documents/sids/sids/693232. pdf.

Ponnamma, S.U., and Manjunath, K. (2012). GC-MS analysis of phytocomponents in the methanolic extract of Justicia wynaadensis (Nees) T. Anders. Int. J. Pharm. Bio. Sci., 3(3): 570 - 576. 
Phytochemical Characterization of Alternanthera sessilis and Assessment of its Growth Promoting Potential on the Freshwater Prawn Macrobrachium rosenbergii

Pourmoghim, H., Haghighi, M., and Rohani, M.S. (2015). Effect of dietary inclusion of Origanum vulgare extract on nonspecific immune responses and hematological parameters of Rainbow trout (Oncorhynchus mykiss). Bull. Environ. Pharma. Life Sci., 4 (3): 33-39.

Radhakrishnan, E.V., Deshmukh, V.D., Maheswarudu, G., Josileen, J., Dineshbabu, A.P., Philipose, K.K., Sarada, P.T., Lakshmi Pillai, S., Saleela, K.N., Chakraborty, R., Dash, G., Sajeev, C.K., Thirumilu, P., Sridhara, B., Muniyappa, Y., Sawant, A.D., Vaidya, N.G., Dias Johny, R., Verma, J.B., Baby, P.K., Unnikrishnan, C., Ramachandran, N.P., Vairamani, A., Palanichamy, A., Radhakrishnan, M., Raju, B., 2012. Prawn fauna (Crustacea: Decapoda) of India - an annotated checklist of the Penaeoid, Sergestoid, Stenopodid and Caridean prawns. Mar. Ass. India, 54, 50-72.

Radhakrishnan, S., Bhavan, P.S., Seenivasan, C., Shanthi, R., and Poongodi, R. (2013). Influence of medicinal herbs (Alteranthera sessilis, Eclipta alba and Cissus quadrangularis) on growth and biochemical parameters of the freshwater prawn Macrobrachium rosenbergii. Aqua. Int., 22: 551-572.

Rajkumar, G., Bhavan, P.S., Srinivasan, V., Udayasuriyan, R., Karthik, M., and Satgurunathan, T. (2017). Partial Replacement of Fishmeal with Marine Algae Turbinaria ornata and Gracilaria corticata for Sustainable Culture of the Freshwater Prawn Macrobrachium rosenbergii. Int. J. Res. Stud. Zool. 3(2): 3244.

Rebecca, A.A., and Bhavan, P.S. (2011). Growth performance of Macrobrachium rosenbergii post larvae fed with vegetable wastes and palmolein supplemented formulated feeds. Rec. Res. Sci. Tech., 3(10): 69-76.

Roe, J.H. (1955). The determination of sugar and blood and spinal fluid with anthrone reagent. J. Biol. Chem., 212: 335-343.

Sambhu, C., Jayaprakas, V. (2001). Livol (IHF-1000), a new herbal growth promoter in white prawn, Penaeus indicus (Crustacea). Ind. J. Mar. Sci., 30: 38-43.

Shabi, M.M., Gayathri, K., Venkatalakshmi, R., and Sasikala, C. (2010). Chemical constituents of hydro alcoholic extract and phenolic fraction of Cynodon dactylon. Int. J. Chem Tech Res., 2(1): 149-154.

Shanthi, R., Bhavan, P.S., and Radhakrishnan, S. (2012). Influence of medicinal herbs, (Andrographis paniculata, Cissus quadrangularis and Eclipta alba) on growth, digestive enzymes, biochemical constituents and protein profile of the freshwater prawn Macrobrachium rosenbergii, Elix. Bio Tech., 42: 6478-6484.

Sivakumar, R., and Sunmathi, D. (2016). Phytochemical screening and antimicrobial activity of ethanolic leaf extract of Alternanthera sessilis (L.) R. BR. EX DC and Alternanthera philoxeroides (MART.) griseb. Eur. J. Pharma., 3(3), 409-412.

Tekinay, A.A., Davies, S.J. (2001). Dietary carbohydrate level influencing feed intake, nutrient utilization and plasma glucose concentration in the rainbow trout, Oncorhynchus mykiss. Turk. J. Vet. Ani. Sci., 25: 657666.

Trease, G.E., and Evans, W.C. (1989). Pharmacology 11th Edn., Bailliere Tindall Ltd., London, 60-75.

Vandendool, H., and Kratz, P.D. (1963) A generalization of the retention index system including linear temperature programmed gas-liquid partition chromatography, J. Chromatogr, 11: 463-471.

Vinodh, K.S.L., and Senthil Kumar, B. (2014). GC-MS Analysis of Aqueous Leaf Extract of Alternantera sessilis. Int. J. Pharma. Res. Schol., 3(1): 382-387.

WebMD (2016). Drugs \& Medications Doxylamine succinate. http://www.webmd.com/drugs/2/drug14124/doxylamine-succinate-oral/details

Citation: R. Kanagarasu et al., "Phytochemical Characterization of Alternanthera sessilis and Assessment of its Growth Promoting Potential on the Freshwater Prawn Macrobrachium rosenbergii ", International Journal of Research Studies in Zoology, vol. 3, no. 4, p. 25-38, 2017. http://dx.doi.org/10.20431/2454-941X.0304004

Copyright: (C) 2017 Authors. This is an open-access article distributed under the terms of the Creative Commons Attribution License, which permits unrestricted use, distribution, and reproduction in any medium, provided the original author and source are credited. 